\title{
Bidirectional asymmetry in the neurovisceral communication for the cardiovascular control: New insights
}

\author{
${ }^{1}$ Prieto I, ${ }^{1}$ Segarra AB, ${ }^{2}$ Martinez-Canamero M, ${ }^{3}$ De Gasparo M, ${ }^{4}$ Zorad S, ${ }^{1}$ Ramirez-Sanchez M \\ ${ }^{1}$ Unit of Physiology, University of Jaen, Jaen, Spain \\ ${ }^{2}$ Unit of Microbiology, University of Jaen, Jaen, Spain \\ ${ }^{3}$ Cardiovascular \& Metabolic Syndrome Adviser, Rossemaison, Switzerland \\ ${ }^{4}$ Institute of Experimental Endocrinology, Biomedical Research Centre of the Slovak Academy of Sciences, Bratislava, \\ Slovakia \\ E-mail:msanchez@ujaen.es
}

\begin{abstract}
The cardiovascular control involves a bidirectional functional connection between the brain and heart. We hypothesize that this connection could be extended to other organs using endocrine and autonomic nervous systems (ANS) as communication pathways. This implies a neuroendocrine interaction controlling particularly the cardiovascular function where the enzymatic cascade of the renin-angiotensin system (RAS) plays an essential role. It acts not only through its classic endocrine connection but also the ANS. In addition, the brain is functionally, anatomically, and neurochemically asymmetric. Moreover, this asymmetry goes even beyond the brain and it includes both sides of the peripheral nervous and neuroendocrine systems. We revised the available information and analyze the asymmetrical neuroendocrine bidirectional interaction for the cardiovascular control. Negative and positive correlations involving the RAS have been observed between brain, heart, kidney, gut, and plasma in physiologic and pathologic conditions. The central role of the peptides and enzymes of the RAS within this neurovisceral communication, as well as the importance of the asymmetrical distribution of the various RAS components in the pathologies involving this connection, are particularly discussed. In conclusion, there are numerous evidences supporting the existence of a neurovisceral connection with multiorgan involvement that controls, among others, the cardiovascular function. This connection is asymmetrically organized.
\end{abstract}

Key words: neuroendocrine interaction, asymmetry, blood pressure, renin-angiotensin system, neuropeptidases, angiotensinases

More than 150 years ago, Claude Bernard already proposed a bidirectional neurovisceral integration between the brain and heart: "Consequently, heart and brain find themselves in an interdependence of mutual intimated action, which is multiplied and reinforced as the organism is growing in sophistication and developing" (Bernard 1878). Recently, this concept was extended and interpreted as a neurovisceral integration model (NIM) (Thayer and Lane 2009). Each individual has a distinctive variability between the beats that make up the heart rate (HRV). Thayer and Lane (2009) have proposed that the HRV that is controlled by the autonomic nervous system (ANS) could be not only an index of the cardiac function but also an index of the degree to which the brain, as an integrating center, generates an adaptive peripheral body's response to environmental changes.

The present review is a compilation that integrates the main data regarding neurovisceral interactions with the data supporting that such interactions may 
be bidirectionally organized in an asymmetrical way. Those neurovisceral interactions are well known but the mechanisms underlying such as communications are not understood. Clearly, the asymmetry in brain functions may determine the bidirectional asymmetric communications. However other mechanisms, for example asymmetries in paired peripheral organs including adrenals, kidneys or gonads, and also in the bilateral autonomic innervation of odd organs such as heart, may be involved in the bidirectional asymmetric organization of the information transfer.

The prefrontal cortex and subcortical areas such as the amygdala form a reciprocal inhibitory circuit (Shoemaker and Goswami 2015), the function of which is to regulate the behavioral and emotional responses to adapt the body to environmental demands and control the function of the ANS affecting the heart and other organs (Williams et al. 2015). These authors have suggested that this circuit could serve as a connection (mediated by the ANS) between the emotional and physiological processes and that its level of activity could be monitored by the HRV. They have proposed that the HRV represents a psychophysiologic index regulated by the ANS and associated with the emotional control capability predicting emotional difficulties (Williams et al. 2015).

However, the concept of the neurovisceral integration could be extended including especially the heart and kidney (Prieto et al. 2014a), adrenals (Prieto et al. 1998), blood (Segarra et al. 2012, 2016), and even gut with its microbiota (Jose and Raj 2015; Grenham et al. 2011), all being involved in the cardiovascular control (CVC). Such a large connection would mainly involve the ANS and the endocrine system. This connection implicates a bidirectional neuroendocrine interaction between the brain and peripheral organs regulating the vital physiologic processes for survival such as glucose metabolism or CVC (Prieto et al. 2014a; McCraty and Shaffer 2015; Ramsay and Woods 2014), as well as a coordinated global response under stress. This integrative response would involve interactions between different central areas, such as prefrontal cortex (PFC), amygdala (AM) or hippocampus (HC) (Hernandez et al. 2015), as well as bidirectional interactions between some of these areas and peripheral tissues (Segarra et al. 2013) or blood (Segarra et al. 2012, 2016), in which the renin-angiotensin system (RAS) would play a major role.

\section{The RAS in the neurovisceral communication}

The RAS plays a key role in the bidirectional integration for CVC. In this system (outlined in Figure 1)
(Ramirez-Sanchez et al. 2013), several aminopeptidases, generically called angiotensinases, play the major role in the metabolism of the various angiotensin (Ang) peptides. The study of their regulation has become an important line of research not only to better understand the function of the RAS but also develop new diagnostic and therapeutic tools for cardiovascular diseases using activators or inhibitors of such enzymes (Gao et al. 2014).

The RAS is crucial in the homeostatic control of body processes. Particularly, brain Ang II (Saavedra 2012) facilitates the stress response whereas other component such as Ang 1-7 or Ang IV, inhibit that reaction (Fontes et al. 2016). The homeostatic response depends on the analysis of the sensory information by central areas to elaborate a motor response conveyed by the ANS in order to quickly get effective control of the target organs. ANS disturbances lead to cardiovascular disability as severe as it can even cause sudden death (Soros and Hachinski 2012). The ANS control depends not only on the brainstem function but also on afferent signals that come from the cortical and limbic areas (Shoemaker et al. 2015).

The kidney contains sensory afferent and efferent sympathetic fibers (Booth et al. 2015). Changes in sympathetic activity modulate the renal mechanisms causing hypertension (Grisk 2005). In heart failure $(\mathrm{HF})$, there is a cardiac and renal efferent sympathetic activation. However, while the efferent sympathetic activity clearly increased renal vasoconstriction, renin release, and sodium retention, the sensory afferent activity has been poorly studied. In fact, the beneficial effects of renal denervation in HF are unclear: it could be due to a denervation of either an efferent sympathetic component or alteration of the sensitive afferent pathway or also a combination of both (Booth et al. 2015). Experimental studies in favor of a renal sensory afferent activation have been reported by Xu et al. (2012) who have observed that rostral ventrolateral medulla projecting neurons to paraventricular nucleus were more active in rats with chronic HF leading to sympathetic nerve activation (SNA). In addition, the inhibitory reno-renal reflex is blunted in HF, which may also stimulate SNA (Kopp et al. 2003).

Clearly, the RAS plays an essential role in the cardiovascular and renal function and its study has revealed new surprising results suggesting its participation in the mentioned bidirectional neurovisceral integration for CVC. Prieto et al. (2014a) have studied the influence of L-NAME (sympathetic stimulation) or propranolol (sympathetic blockade) treatment on angiotensinase activities in the hypothalamus $(\mathrm{HT})$, left ventricle (VT), renal cortex (RC), and re- 


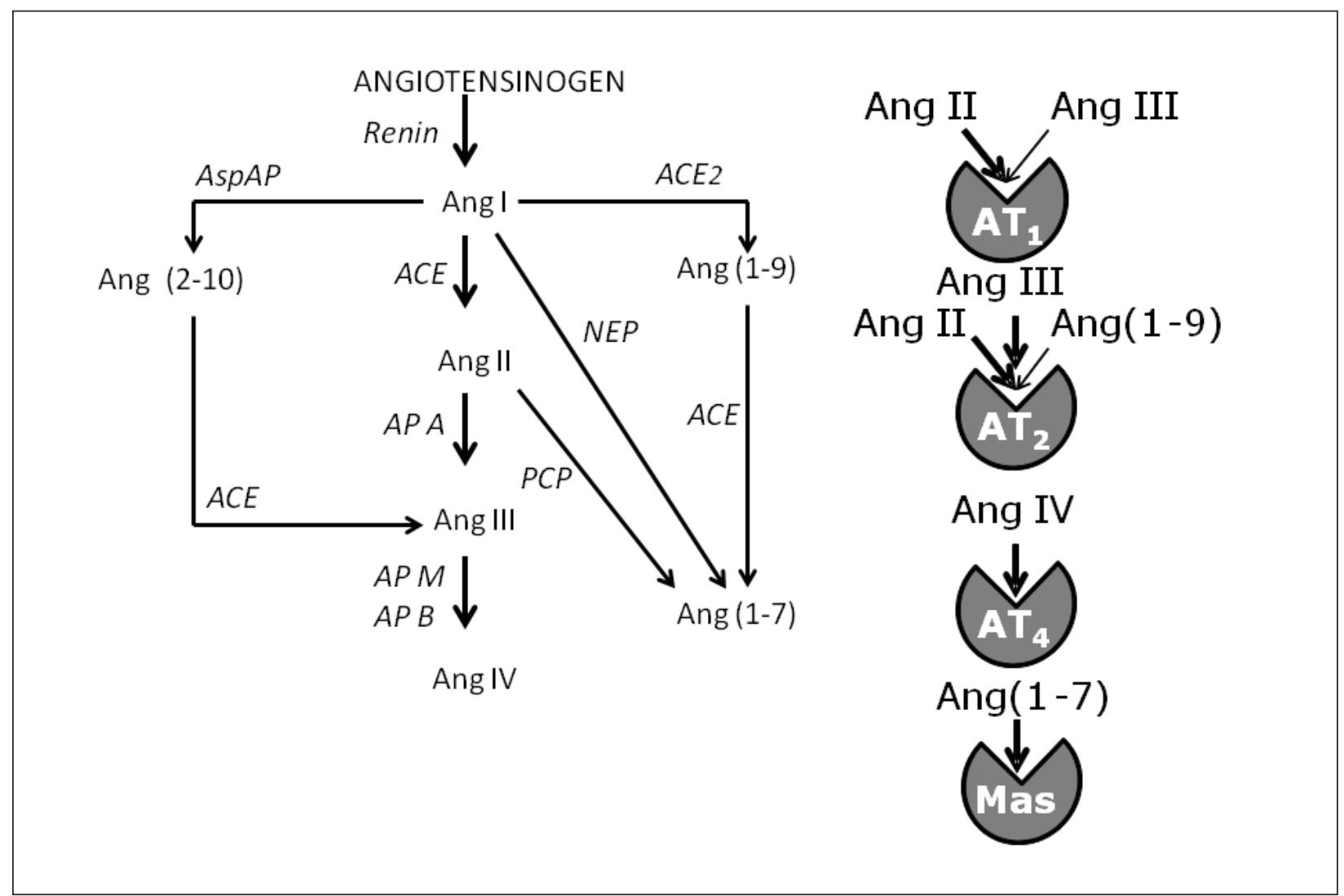

Figure 1. The proteolytic enzymes (angiotensinases) of the renin-angiotensin system (RAS) are responsible for generating different peptides with different functions. Angiotensin (Ang) I (1-10) is produced by the activity of renin on its inactive precursor Angiotensinogen. The angiotensin converting enzyme (ACE) is responsible for the Ang II (Ang 1-8) formation which is further metabolized to Ang III (Ang 2-8) by glutamyl aminopeptidase (AP A). Alternatively, Ang I may be metabolized to other active peptides depending on the enzyme involved. Aspartyl-aminopeptidase (AspAP) hydrolyzes the Asp amino-terminal to produce Ang 2-10, which could be further converted to Ang III by the action of ACE. Furthermore, Ang I may be converted to Ang 1-7 by neutral endopeptidase (NEP) or to Ang 1-9 by the ACE homologous ACE Ang 1-9 could be further converted to Ang 1-7 by ACE. Ang III could be metabolized to Ang IV (Ang 3-8) through the action of either arginyl-aminopeptidase (AP B) or alanyl-aminopeptidase (AP M). Ang IV is further converted to Ang 4-8 again by the action of AP M and then converted to Ang 5-8 by the activity of the adipocytederived leucine aminopeptidase (A-LAP). The $\mathrm{AT}_{1}$ receptor binds essentially Ang II but also Ang III with a lower affinity; the $\mathrm{AT}_{2}$ receptor binds mostly Ang III and Ang II but also Ang 1-9; the $\mathrm{AT}_{4}$ receptor binds specifically Ang IV whereas the Mas-receptor binds exclusively Ang 1-7 (Ramirez-Sanchez et al. 2013). The thickness of the arrow indicates the degree of affinity for the receptor.

nal medulla (RM) of normotensive Wistar-Kyoto and spontaneously hypertensive rats (SHR). Results have revealed a high incidence of highly significant correlations between angiotensinase activities when these tissues were compared. The correlations observed in such studies not only allowed to discriminate between the activation of the sympathetic or parasympathetic systems but also hypothesize the participation of the angiotensinases and consequently the RAS in the mechanisms that bidirectionally connect brain, heart, and kidney for CVC. In SHR, sympathetic hyperactivation with a negative relationship of angiotensinases between HT and VT and a positive one between HT and RC could be suggested after
L-NAME treatment (Figure 2). It should be noted that these relationships could not only involve the classical endocrine RAS connection but also other types of communication systems with the various components of the RAS, which could travel bidirectionally through the ANS. It has been hypothesized that the mechanisms, by which this communication takes place, could involve retro-control processes not yet clearly identified. For example, the existence of a bidirectional axonal transport involving various components of the RAS such as angiotensin-converting enzyme (Kato et al. 1987; Aiso et al. 1988), Ang II binding sites through the vagus nerve (Diz and Ferrario 1988) or angiotensinases (Prieto et al. 2014a) 


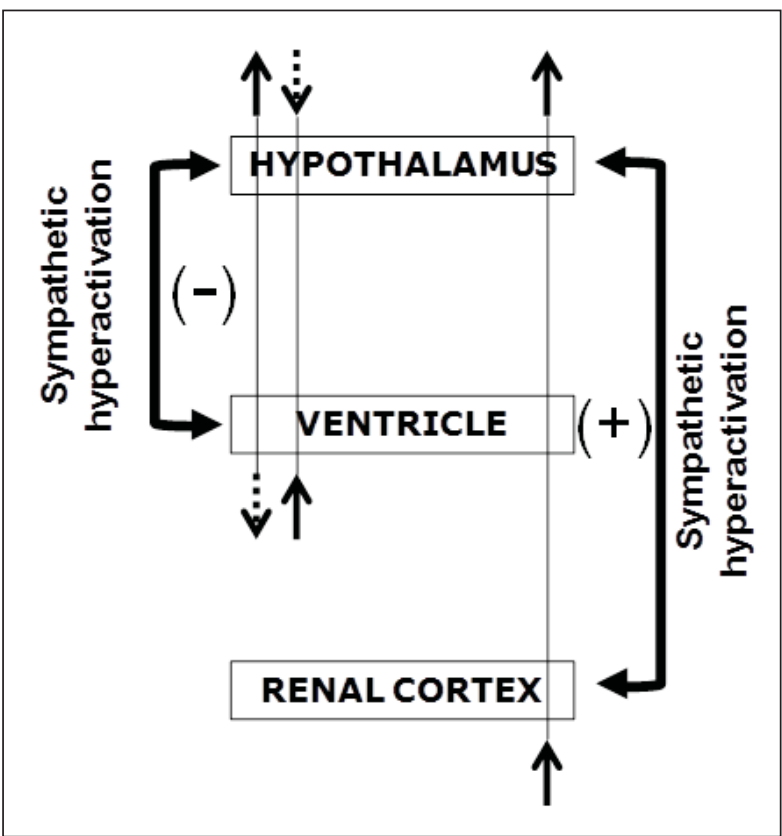

Figure 2. Functional state of the renin-angiotensin system (RAS), reflected by the activity of the enzymes involved in its cascade (angiotensinases), in a model of essential hypertension with sympathetic hyperactivation. (-) indicates a negative correlation between hypothalamus and ventricle. $(+)$ indicates a positive correlation between renal cortex and hypothalamus. The continuous arrows show an increased turnover of RAS and the discontinuous one a decreased one. When the hypothalamic RAS turnover is increased, the ventricular is decreased and vice versa, when the ventricular RAS turnover is increased, the hypothalamic is decreased. In contrast, when the hypothalamic or renal RAS turnover is increased, the renal or hypothalamic will be too (Prieto et al. 2014a).

has been suggested. Furthermore, it has been hypothesized that the ANS could participate in the regulation of secretory mechanisms for angiotensinases in the neuro-effector junctions, either directly from the efferent nerve terminals or secreted from the tissues themselves under their activation through autonomic nerve terminals (Prieto et al. 2014a; Banegas et al. 2009). Afferent neurons could also carry angiotensinases from target tissues to brain (Prieto et al. 2014a) (Figure 3). Because the cardiac and renal effects observed in the patients with essential hypertension have been faithfully reproduced in L-NAME treated hypertensive animals (Zhou and Frohlich 2007), the high levels of correlation observed by Prieto et al. (2014a) among brain, heart, and kidney may reflect to some extend the mechanisms associated with the severity of the cardio-renal damage of such patients. These results could add valid information to clarify the afferent and efferent mechanisms involved in

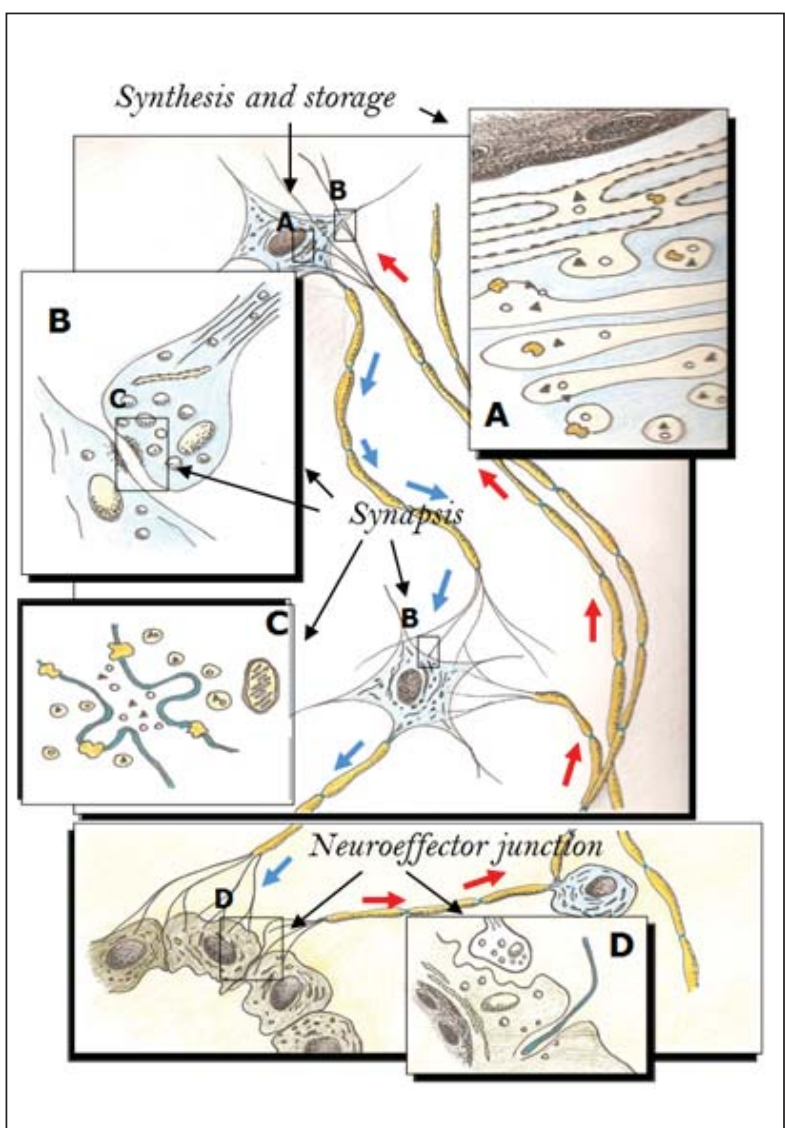

Figure 3. Drawing of a hypothetical model of neurovisceral integration in which, the renin-angiotensin system (RAS) would be involved through the autonomic nervous system (ANS) (Prieto et al. 2014a). (A) The peptidergic precursors and proteolytic enzymes, synthesized by ribosomes of the rough endoplasmic reticulum, are moved through the cisterns of the reticulum until the Golgi apparatus where they will be stored, either coexisting or independently in secretory granules that will be transported through the axon, where presumably their processing take place by the action of proteases which, could also be co-transported in the same vesicles. (B), (C) and (D) Once in the nerve terminal or neuroeffector junction, through a calcium-dependent mechanism, the vesicular content is released into the inter-synaptic or interstitial space. Coming up next, the peptide-receptor (post and/or presynaptic) interaction take place, thus leading to the cellular effect. Once carried out its action, the peptide will be inactivated or removed from the interstitial or inter-synaptic space through degradation by soluble or membrane-bound peptidases, by synaptic recapture or by internalization of the peptide-receptor complex with subsequent fusion of vesicles with enzymatic content (Hallberg 2015). The functional status of angiotensins and angiotensinases in the neuroendocrine axis could be regulated by the ANS through feedback mechanisms not yet clearly established. It has been suggested and described, an anterograde and retrograde axonal transport for several components of the RAS such as angiotensinases, angiotensin II and its receptor (Prieto et al. 2014; Kato et al. 1987; Aiso et al. 1988; Diz and Ferrario 1988). In addition, the ANS could regulate secretory processes for angiotensinases in the neuroeffector junctions, either from nerve terminals or from the tissues themselves (Prieto et al. 2014). 
cardio-renal damage in hypertensive subjects which may lead to HF. This clearly implies the brain-heartkidney axis (Booth et al. 2015). Angiotensinases are involved in the metabolism of the different angiotensin peptides, inactivating and transforming them in new peptides with different functions and properties (Figure 1) (Ramirez-Sanchez et al. 2013). Therefore, the degree of these activities reflects the level of the angiotensin substrates and the resulting biotransformed products.

Therefore, we could speculate that the sympathetic hyperarousal in L-NAME treated hypertensive animals may lead to a parallel higher turnover (higher angiotensinase activities) of Angs in HT and RC, the functional status of both local hypothalamic and renal RAS being connected together via the sympathetic branch of the ANS (Prieto et al. 2014a). However, the sympathetic hyperactivation in L-NAME treated rats would simultaneously lead to an inverse turnover of angiotensin peptides between the hypothalamus and the heart, whereas the angiotensinase activity increases in the hypothalamus and decreases in the heart (Figure 2). This may reflect an imbalance of the functional status of the RAS between the hypothalamus and the heart and between the hypothalamuskidney and the hypothalamus-heart axes in the specific conditions following L-NAME treatment in SHR.

\section{The gut in the neuroendocrine integration for the cardiovascular control}

For CVC, there are integrative mechanisms that functionally connect not only the brain, heart or kidney, but also other tissues such as the gut and even its microbiota. For that reason, there are currently intense investigations on the role played by dietary factors, especially fats, in CVC (Michas et al. 2014; Villarejo et al. 2015). The composition of the gut microbiota, conditioned by the type of diet, could hypothetically influence the brain function and participate in CVC not only through neural brainheart connections but also through factors produced by both the microbiota and the intestine (Ito and Adachi-Akahane 2013). Thus, different types of diets differentially modify the gut microbiota (Hidalgo et al. 2014), which influences the brain function (Grenham et al. 2011). Moreover, different types of diets differentially influence the cardiovascular function (involving the RAS) (Villarejo et al. 2015) and the profile of fatty acids and neuropeptides (including Angs) in the frontal cortex (Segarra et al. 2011) affecting the cortical functions. In fact, the absence of gut microbiota radically alters the nervous and neuroen- docrine responses to stress (Crumeyrolle-Arias et al. 2014), suggesting a functional bidirectional relationship between the gut and the brain neuropeptides (Holzer and Farzi 2014). This involves brain areas such as the hypothalamus, cortex and the limbic system (Bonaz 2013). The abundance of Firmicutes and Bacteroidetes in the gut microbiota is associated with an increased blood pressure in several models of hypertension and decreased gut microbiota, following antibiotics treatments, may modulate blood pressure (BP) (Jose and Raj 2015). However, to our knowledge, there are still no reports demonstrating direct effect of the gut microbiota and diet on CVC. Preliminary results have suggested, however, such influence (Prieto et al. 2014b). These authors have compared a diet enriched with butter with another supplemented with virgin olive oil. Whereas high butter diet produced a greater accumulation of visceral adipose tissue and higher BP levels, the diet supplemented with virgin olive oil was associated with lower levels of triglycerides in plasma, higher HDL/LDL ratio and lower BP. These values correlated with the percentage of selective microbial groups suggesting that the beneficial effects of virgin olive oil were linked to modulation of intestinal microbiota.

All these observations could be interlinked. The type of diet could modify the intestinal microbiota and influence the brain and cardiovascular function in an integrated manner in which autonomic and endocrine systems participate. All this falls within the scope of the neurovisceral integration, in which the nervous system, whose development can be influenced by the intestinal microbiota (Kabouridis and Pachnis 2015), and the enteric endocrine system, including the classical APUD (amine precursor uptake and decarboxilation) system, could play a major role. Pearse and Polak (1971) proposed that APUD cells, where classic neurotransmitters and neuropeptides coexist, are originated from neural crest and interact with both autonomous and endocrine systems (Baylis et al. 1993). Neuropeptides from cerebral and intestinal origin might be part of a functional unit that plays an important role in the establishing a bidirectional control (through ANS and endocrine system) between the involved tissues. Some neuropeptides involved are: substance $\mathrm{P}$, neuropeptide $\mathrm{Y}$, vasoactive intestinal polypeptide, somatostatin (Holzer and Farzi 2014), cholecystokinin (Dockray 2013) or Angs (Fandriks 2011). The mechanisms by which these neuropeptides may connect bilaterally gut and brain not only include endocrine pathways and the stimulation of afferent and efferent neurons (Holzer and Farzi 2014) but also axonal transport of factors 
that influence both gut and brain. In fact, this type of connection is possible as vagal axonal transport of RAS components such as angiotensin II binding sites (Diz and Ferrario 1988), as well as factors such as $\alpha$-synuclein has been observed directly from gut to brain (Holmqvist et al. 2014).

Finally, if the ANS works asymmetrically, it is presumable that this asymmetry also implies gut functions and some evidences may support this hypothesis. Indeed, a significant negative association has been observed between left-handedness and early satiation (Fujiwara et al.2011) and a positive one with inflammatory bowel diseases (Morris et al. 2001), in which Ang II is also involved (Fandriks 2011).

\section{Brain asymmetry}

The available data have shown that the asymmetry extends to all organizational levels of the main internal communication systems: brain structures, peripheral nervous system, and endocrine glands (Ramirez et al. 2004). Thus, one can consider neuropeptides in the brain structures and their regulatory enzymes (neuropeptidases, including angiotensinases). In the brain, there are asymmetries in the content of gonadotropin-releasing hormone that predominates in the right hypothalamus (Gerendai and Halasz 2001). Also, thyrotropin-releasing hormone prevails in the left hypothalamus (Borson-Chazot et al. 1986). Furthermore, cholecystokinin- 8 or angiotensin II facilitate learning and memory when microinjected respectively into the left amygdala (Belcheva et al. 1994) or hippocampus (Tashev and Stefanova 2015). Several angiotensinases also show significant inter-hemispheric differences in amygdala, hippocampus, and prefrontal cortex (Banegas et al. 2005). Considering the peripheral nervous system and endocrine glands, one also observed a lateralization in reproductive functions where right brain structures apparently play a predominant role: indeed, right but not left vagotomy of testes and ovaries lead to increased levels in serum luteinizing hormone (Gerendai and Halasz 2001). In addition, it was reported that right, but not left prefrontal cortex, lesions decreased corticosterone levels in acutely stressed animals suggesting right hemisphere dominance in the control of the hypothalamic-pituitary-adrenal axis (Sullivan and Gratton 1999). This involves a lateralization of the innervation of the paired endocrine glands, gonads, and adrenals, via the vagus nerve, which confer a key role to the right hemisphere in the control of autonomic functions in order to increase the chances of the subject survival (Gerendai and Halasz 2001).
Finally, indirect data also suggest that angiotensinase activities act asymmetrically in the control of BP and water balance and this also involves the ANS (Prieto et al. 2014a). Moreover, such asymmetry is not static but dynamic and adjustable depending on the variability in exogenous conditions such as light or dark conditions (Ramirez et al. 2004) or endogenous environment like type and level of sex steroids (Ramirez et al. 2008; Rueda et al. 2016), stress (Lee et al. 2014), brain dopamine content (Banegas et al. 2009, 2011) or drug treatments involving the RAS, such as ACE inhibitors (Segarra et al. 2012, 2013).

\section{Asymmetry in the neuroendocrine interaction for the cardiovascular control}

This section describes the major available data that may support a bidirectional asymmetry in the neurovisceral integration model for CVC. Thus, stroke affecting the left or right hemisphere is a major cause of disability and death (Rodgers 2013). Its prognosis is very serious and an early appropriate evaluation is essential to establish an effective treatment. Stroke usually involves alterations of the ANS (Klingelhofer and Sander 1997). Physiologically, there is a neuroendocrine asymmetry that includes the ANS (Ramirez et al. 2004; Gerendai and Halasz 2001). Studies in animals and humans have suggested that the generated disturbance is depending on the brain area and the injured side.

These studies have highlighted the importance of the asymmetric organization of both central and peripheral nervous system in CVC. However, the results are confusing and sometimes apparently contradictory. Patients with lateralized neurological disorders develop myocardial problems. The pathogenesis of these disorders is still unknown and various hypotheses have been proposed. For example, a lateralized sympathetic activation with right predominance has been suggested after a right hemisphere stroke with increased serum levels of norepinephrine and cardiac arrhythmias (Sander and Klingelhofer 1995). In animal models, insular lesions may cause cardiac arrhythmias. Something similar may happen in humans. While right insular stimulation activates the sympathetic cardiovascular tone, the left insular stimulation activates the parasympathetic one (Oppenheimer 2006). It is worth, however to indicate that no clear predominant laterality on CVC or stroke prognosis in relation to insular cortex stroke has been reported in patients (Nagai et al. 2010). On the other hand, under stress, heart disease correlates with an asymmetric hyperactivation of certain brain 
areas such as the left parietal cortex or the right visual association cortex (Soufer et al. 1998). Inducing a stroke in genetically hypertensive rats with a diet containing $4 \% \mathrm{NaCl}$ and $0.75 \%$ of $\mathrm{K}^{+}$, results in higher density of cerebrovascular sympathetic nerves in the left hemisphere than in the right (Smeda 1990). It has also been speculated that parasympathetic tone was reduced ipsilateral to the affected hemisphere linked to an increase in the sympathetic one of the same side (Lane et al. 1992).

Zamrini et al. (1990) have analyzed the heart rate in patients after the unilateral inactivation of hemispheres by intracarotid amobarbital administration. The heart rate increased or decreased after the inactivation of either the left or the right hemisphere, respectively suggesting the existence of anatomical or functional asymmetries in the central and peripheral nervous system. Also, occlusion of the left or right middle cerebral artery in normotensive rats reduces BP only in animals with left occlusion (Hachinski et al. 1992). In these experiments, renal sympathetic nerve discharge increased after left occlusion (suggesting RAS activation) whereas plasma norepinephrine increased after right occlusion.

Additionally, hypertension has been associated with a vascular dementia and cognitive impairment. Chiefly, one observes a direct relationship between the hypertension and atrophy of the left hemisphere in humans (Lis and Gaviria 1997). It has also been noted that systolic BP tended to be higher and less stable after ischemic stroke located mainly in the left hemisphere (Dicker et al. 2006). Studies in mice have suggested that the left cerebral ischemia produces cardiac dysfunction associated with extensive damage of insular cortex (Min et al. 2009). Furthermore, based on anatomical studies of the asymmetrical ways that originate from the dorsomedial nucleus of the left or right hypothalamus (Toga and Thompson 2003), Xavier et al. (2013) studied the possible asymmetry in the cardiovascular responses to the stimulation of that nucleus. Their results have indicated that these pathways are asymmetric and that the right dorsomedial nucleus may exert a predominant control on the cardiovascular function in situations of emotional stress.

Other studies in normotensive and hypertensive rats have indicated a dramatic and progressive increase in BP in animals after left lesions of the nigrostriatal system with left hemisphere dopamine depletion of more than $90 \%$. This was not accompanied by an increase in heart rate suggesting a change in the peripheral resistance rather than a contribution of the cardiac output (Banegas et al. 2011). This again supports a functional asymmetry of the ANS in CVC. Moreover, unilateral lesions of the nigrostriatal system cause a change in plasma angiotensinases and nitric oxide (NO) content following vascular sympathetic activation, which again differs depending on the side of the brain lesion. Angiotensinase activities and $\mathrm{NO}$ concentration showed a negative plasma correlation in the right lesioned normotensive rats and left lesioned hypertensive ones, which also suggests an asymmetric control of the ANS in that response (Banegas et al. 2009). Hypertensive animals treated with the ACE inhibitor captopril showed a marked number of correlations between angiotensinase activities obtained in the right frontal cortex (but not in the left) and the ventricle in comparison with nontreated control animals that fundamentally showed correlations between the ventricle and the left frontal cortex (Segarra et al. 2013). In contrast, the existence of correlations between the plasma angiotensinase activities and right or left frontal cortex are reversed in animals treated or not treated with captopril. While in control animals, the angiotensinase activities correlated significantly with the right frontal cortex, in animals treated with captopril, the correlations were with the left cortex (Segarra et al. 2012). Therefore, alterations of the RAS (i.e. ACE inhibition) asymmetrically modify the neuroendocrine response because they differentially affect both the left or right nervous system-heart axis and the left or right nervous system-blood axis. Rueda et al. (2016) have observed significant differences in BP depending on the gender, handedness, the phase of the ovarian cycle, and the arm, in which the BP was determined. The authors have described higher levels of pressure in left-handers women during the menstrual phase. They have also reported that the level of motor laterality correlated negatively with BP measured in the left arm of right-handers women in ovulatory phase but positively with the pressure measured in the right arm of the right-handers women in menstrual phase.

\section{Conclusions}

There are abundant evidences supporting the existence of a neurovisceral connection with multiorgan involvement that controls, among others, the cardiovascular function. In addition, it is also obvious that the cardiovascular, nervous, and neuroendocrine functions are asymmetrically organized. Therefore, it seems logical to think that this is also true for the neurovisceral integration. Such asymmetry is not only motor, cognitive or emotional but also autonomous and neuroendocrine involving the whole body. 
Although available data does not allow establishing a general pattern for the bidirectional asymmetry in the cardiovascular control, one can speculate about inverse reciprocal interactions between the left and the right brain areas through modulation of the ANS and RAS.

Hypothetically, various types of unilateral lesions at several central and peripheral levels of the nervous system may lead to contralateral compensatory mechanisms that determine the cardiovascular response. Similarly, cardiac abnormalities may determine asymmetries in the central response that would be necessary to know in order to assess the prognosis and treatment not only for the cardiac disorders but also for its cognitive consequences. While multiple asymmetries in physiologic and pathologic conditions have been described, a direct relationship has not yet been observed between the occurrence of a definite asymmetry or its basal modification and the etiology of a given disease. We don't know whether these changes are a cause or a result of the disease. Thus, the search for the systematic patterns of asymmetric behaviors in health and disease will continue.

Further detailed development of our proposal may help in the diagnosis and prognosis of the severity of the pathological entity depending on the brain or the peripheral organ lesion's sides.

\section{Acknowledgements}

This work was supported by the Junta de Andalucia through the excellence project AGR 6340 and by the University of Jaen through the project UJA 091303.

\section{References}

Aiso M, Potter WZ, Saavedra JM. Axonal transport of angiotensin-converting enzyme in the rat striatonigral pathway. Brain Res 447, 195-199, 1988

Banegas I, Prieto I, Alba F, Vives F, Araque A, Segarra AB, Duran R, de Gasparo M, Ramirez M. Angiotensinase activity is asymmetrically distributed in the amygdala, hippocampus and prefrontal cortex of the rat. Behav Brain Res 156, 321-326, 2005.

Banegas I, Prieto I, Vives F, Alba F, de Gasparo M, Duran R, Luna J de D, Segarra AB, Hermoso F, Ramirez M. Asymmetrical response of aminopeptidase $\mathrm{A}$ and nitric oxide in plasma of normotensive and hypertensive rats with experimental hemiparkinsonism. Neuropharmacology 56, 573-579, 2009.

Banegas I. Prieto I, Segarra AB, Duran R, Vives F, Alba F, Luna JD, de Gasparo M, Wangesteen R, Ruiz-Bailen M, Ramirez-Sanchez M. Blood pressure increased dramatically in hypertensive rats after left hemisphere lesions with 6-hydroxydopamine. Neurosci Lett 500, 148-150, 2011.

Baylis BW, Tranmer BI, Ohtaki M. Central and autonomic nervous system links to the APUD system (and their APUDomas). Semin Surg Oncol 9, 387-393, 1993.

Belcheva I, Belcheva S, Petkov VV, Petkov VD. Asymmetry in behavioral responses to cholecystokinin microinjected into rat nucleus accumbens and amygdala. Neuropharmacology 33, 995-1002, 1994.

Bernard Claude. Etude sur la physiologie du coeur. La science experimentale 316-366, 1878.

Bonaz B. Inflammatory bowel diseases: a dysfunction of brain-gut interactions? Minerva Gastroenterol Dietol 59, 241-259, 2013.

Booth LC, May CN, Yao ST. The role of the renal afferent and efferent nerve fibers in heart failure. Front Physiol 6, 270,2015

Borson-Chazot F, Jordan D, Fevre-Montange M, Kopp N, Tourniaire J, Rouzioux JM, Veisseire M, Mornex R. TRH and LH-RH distribution in discrete nuclei of the human hypothalamus: evidence for a left prominence of TRH. Brain Res 382, 433-436, 1986.

Crumeyrolle-Arias M, Jaglin M, Bruneau A, Vancassel S, Cardona A, Dauge V, Naudon L, Rabot S. Absence of the gut microbiota enhances anxiety-like behavior and neuroendocrine response to acute stress in rats. Psychoneuroendocrinology 42, 207-217, 2014.

Dicker D, Maya I, Vasilevsky V, Gofman M, Markowitz D, Beilin V, Sarid M, Yosefy C. Blood pressure variability in acute ischemic stroke depends on hemispheric stroke location. Blood Press 15, 151-156, 2006.

Diz DI, Ferrario CM. Bidirectional transport of angiotensin II binding sites in the vagus nerve. Hypertension 11, I139-I143, 1988

Dockray GJ. Enteroendocrine cell signalling via the vagus nerve. Curr Opin Pharmacol 13, 954-958, 2013.

Fandriks L. The renin-angiotensin system and the gastrointestinal mucosa. Acta Physiol (Oxf) 201, 157-167, 2011. 
Fontes MA, Martins Lima A, Santos RA. Brain angiotensin-(1-7)/Mas axis: A new target to reduce the cardiovascular risk to emotional stress. Neuropeptides 56, 9-17, 2016.

Fujiwara Y, Kubo M, Kohata Y, Yamagami H, Tanigawa T, Watanabe K, Watanabe T, Tominaga K, Arakawa T. Association between left-handedness and gastrointestinal symptoms. Digestion 84, 114-118, 2011.

Gao J, Marc Y, Iturrioz X, Leroux V, Balavoine F, Llorens-Cortes C. A new strategy for treating hypertension by blocking the activity of the brain renin-angiotensin system with aminopeptidase A inhibitors. Clin Sci (Lond) 127, 135-148, 2014.

Gerendai I, Halasz B. Asymmetry of the neuroendocrine system. News Physiol Sci 16, 92-95, 2001.

Grenham S, Clarke G, Cryan JF, Dinan TG. Brain-gut-microbe communication in health and disease. Front Physiol 2, 94, 2011.

Grisk O. Sympatho-renal interactions in the determination of arterial pressure: role in hypertension. Exp Physiol 90 , 183-187, 2005.

Hachinski VC, Oppenheimer SM, Wilson JX, Guiraudon C, Cechetto DF. Asymmetry of sympathetic consequences of experimental stroke. Arch Neurol 49, 697-702, 1992.

Hallberg M. Neuropeptides: metabolism to bioactive fragments and the pharmacology of their receptors. Med Res Rev 35, 464-519, 2015.

Hernandez J, Prieto I, Segarra AB, de Gasparo M, Wangensteen R, Villarejo AB, Banegas I, Vives F, Cobo J, RamirezSanchez M. Interaction of neuropeptidase activities in cortico-limbic regions after acute restraint stress. Behav Brain Res 287, 42-48, 2015.

Hidalgo M, Prieto I, Abriouel H, Cobo A, Benomar N, Galvez A, Martinez-Canamero M. Effect of virgin and refined olive oil consumption on gut microbiota. Comparison to butter. Food Res Int 64, 553-559, 2014.

Holmqvist S, Chutna O, Bousset L, Aldrin-Kirk P, Li W, Bjorklund T, Wang ZY, Roybon L, Melki R, Li JY. Direct evidence of Parkinson pathology spread from the gastrointestinal tract to the brain in rats. Acta Neuropathol 128, 805-820, 2014.

Holzer P, Farzi A. Neuropeptides and the microbiota-gut-brain axis. Adv Exp Med Biol 817, 195-219, 2014.

Ito $\mathrm{M}$, Adachi-Akahane S. Inter-organ communication in the regulation of lipid metabolism: focusing on the network between the liver, intestine, and heart. J Pharmacol Sci 123, 312-317, 2013.

Jose PA, Raj D. Gut microbiota in hypertension. Curr Opin Nephrol Hypertens 24, 403-409, 2015.

Kabouridis PS, Pachnis V. Emerging roles of gut microbiota and the immune system in the development of the enteric nervous system. J Clin Invest 125, 956-964, 2015.

Kato T, Ishihara H, Shimizu A, Yokosawa H, Ishii S, Komiya Y. The axonal transport of dipeptidyl aminopeptidase II, angiotensin-converting enzyme and other peptidases in rat sciatic nerves. Neurosci Res 4, 241-248, 1987.

Klingelhofer J, Sander D. Cardiovascular consequences of clinical stroke. Baillieres Clin Neurol 6, 309-335, 1997.

Kopp UC, Cicha MZ, Smith LA. Impaired responsiveness of renal mechanosensory nerves in heart failure: role of endogenous angiotensin. Am J Physiol Regul Integr Comp Physiol 284, R116-R124, 2003.

Lane RD, Wallace JD, Petrosky PP, Schwartz GE, Gradman AH. Supraventricular tachycardia in patients with right hemisphere strokes. Stroke 23, 362-366, 1992.

Lee SW, Gerdes L, Tegeler CL, Shaltout HA, Tegeler CH. A bihemispheric autonomic model for traumatic stress effects on health and behavior. Front Psychol 5, 843, 2014.

Lis CG, Gaviria M. Vascular dementia, hypertension, and the brain. Neurol Res 19, 471-480, 1997.

McCraty R, Shaffer F. Heart Rate Variability: New Perspectives on Physiological Mechanisms, Assessment of Selfregulatory Capacity, and Health risk. Glob Adv Health Med 4, 46-61, 2015.

Michas G, Micha R, Zampelas A. Dietary fats and cardiovascular disease: putting together the pieces of a complicated puzzle. Atherosclerosis 234, 320-328, 2014.

Min J, Farooq MU, Greenberg E, Aloka F, Bhatt A, Kassab M, Morgan JP, Majid A. Cardiac dysfunction after left permanent cerebral focal ischemia: the brain and heart connection. Stroke 40, 2560-2563, 2009.

Morris DL, Montgomery SM, Galloway ML, Pounder RE, Wakefield AJ. Inflammatory bowel disease and laterality: is left handedness a risk? Gut 49, 199-202, 2001.

Nagai M, Hoshide S, Kario K. The insular cortex and cardiovascular system: a new insight into the brain-heart axis. J Am Soc Hypertens 4, 174-182, 2010.

Oppenheimer S. Cerebrogenic cardiac arrhythmias: cortical lateralization and clinical significance. Clin Auton Res 16, 6-11, 2006.

Pearse AG, Polak JM. Neural crest origin of the endocrine polypeptide (APUD) cells of the gastrointestinal tract and pancreas. Gut 12, 783-788, 1971. 
Prieto I, Martinez A, Martinez JM, Ramirez MJ, Vargas F, Alba, F, Ramirez M. Activities of aminopeptidases in a rat saline model of volume hypertension. Horm Metab Res 30, 246-248, 1998.

Prieto I, Villarejo AB, Segarra AB, Banegas I, Wangensteen R, Martinez-Canamero M, de Gasparo M, Vives F, Ramirez-Sanchez M. Brain, heart and kidney correlate for the control of blood pressure and water balance: role of angiotensinases. Neuroendocrinology 100, 198-208, 2014a.

Prieto I, Hidalgo M, Cobo A, Segarra AB, Ramirez M, Galvez A, Vives F, Martinez-Canamero M. BAM-PTH1038 -Comparative study of the effect of virgin olive oil and butter on the intestinal microbiota and diverse parameters related to metabolic syndrome. XIVth International Congress of Bacteriology and Applied Microbiology. Montreal, Canada, International Union of Microbiological Societies Congresses 1268, $2014 \mathrm{~b}$.

Ramirez M, Prieto I, Vives F, de Gasparo M, Alba F. Neuropeptides, neuropeptidases and brain asymmetry. Curr Protein Pept Sci 5, 497-506, 2004.

Ramirez M, Prieto I, Alba F, Vives F, Banegas I, de Gasparo M. Role of central and peripheral aminopeptidase activities in the control of blood pressure: a working hypothesis. Heart Fail Rev 13, 339-353, 2008.

Ramirez-Sanchez M, Prieto I, Wangensteen R, Banegas I, Segarra AB, Villarejo AB, Vives F, Cobo J, de Gasparo M. The renin-angiotensin system: new insight into old therapies. Curr Med Chem 20, 1313-1322, 2013.

Ramsay DS, Woods SC. Clarifying the roles of homeostasis and allostasis in physiological regulation. Psychol Rev 121, 225-247, 2014.

Rodgers H. Stroke Handb Clin Neurol 110, 427-433, 2013.

Rueda I, Banegas I, Prieto I, Wangensteen R, Segarra AB, Villarejo AB, De Gasparo M, Luna JD, Vives F, RuizBailen M, Ramirez-Sanchez M. Handedness and gender influence blood pressure in young healthy men and women: A pilot study. Endocr Regul 50, 10-15, 2016.

Saavedra JM. Angiotensin II AT(1) receptor blockers as treatments for inflammatory brain disorders. Clin Sci (Lond) 123, 567-590, 2012.

Sander D, Klingelhofer J. Changes of circadian blood pressure patterns and cardiovascular parameters indicate lateralization of sympathetic activation following hemispheric brain infarction. J Neurol 242, 313-318, 1995.

Segarra AB, Ruiz-Sanz JI, Ruiz-Larrea MB, Ramirez-Sanchez M, de Gasparo M, Banegas I, Martinez-Canamero M, Vives F, Prieto I. The profile of fatty acids in frontal cortex of rats depends on the type of fat used in the diet and correlates with neuropeptidase activities. Horm Metab Res 43, 86-91, 2011.

Segarra AB, Prieto I, Banegas I, Villarejo AB, Wangensteen R, de Gasparo M, Vives F, Ramirez-Sanchez M. Asymmetrical effect of captopril on the angiotensinase activity in frontal cortex and plasma of the spontaneously hypertensive rats: expanding the model of neuroendocrine integration. Behav Brain Res 230, 423-427, 2012.

Segarra AB, Prieto I, Banegas I, Villarejo AB, Wangensteen R, de Gasparo M, Vives F, Ramirez-Sanchez M. The brain-heart connection: frontal cortex and left ventricle angiotensinase activities in control and captopriltreated hypertensive rats-a bilateral study. Int J Hypertens 2013:156179, 2013.

Segarra AB, Hernandez J, Prieto I, de Gasparo M, Ramirez-Sanchez M. Neuropeptidase activities in plasma after acute restraint stress. Interaction with cortico-limbic areas. Acta Neuropsychiatr 28, 239-243, 2016.

Shoemaker JK, Goswami R. Forebrain neurocircuitry associated with human reflex cardiovascular control. Front Physiol 6, 240, 2015.

Shoemaker JK, Norton KN, Baker J, Luchyshyn T. Forebrain organization for autonomic cardiovascular control. Auton Neurosci 188, 5-9, 2015.

Smeda JS. Analysis of cerebrovascular sympathetic nerve density in relation to stroke development in spontaneously hypertensive rats. Stroke 21, 785-789, 1990.

Soros P, Hachinski V. Cardiovascular and neurological causes of sudden death after ischaemic stroke. Lancet Neurol 11, 179-188, 2012.

Soufer R, Bremner JD, Arrighi JA, Cohen I, Zaret BL, Burg MM, Goldman-Rakic, P. Cerebral cortical hyperactivation in response to mental stress in patients with coronary artery disease. Proc Natl Acad Sci U S A 95, 6454-6459, 1998.

Sullivan RM, Gratton A. Lateralized effects of medial prefrontal cortex lesions on neuroendocrine and autonomic stress responses in rats. J Neurosci 19, 2834-2840, 1999.

Tashev R, Stefanova M. Hippocampal asymmetry in angiotensin II modulatory effects on learning and memory in rats. Acta Neurobiol Exp (Wars) 75, 48-59, 2015.

Thayer JF, Lane RD. Claude Bernard and the heart-brain connection: further elaboration of a model of neurovisceral integration. Neurosci Biobehav Rev 33, 81-88, 2009.

Toga AW, Thompson PM. Mapping brain asymmetry. Nat Rev Neurosci 4, 37-48, 2003. 
Villarejo AB, Ramirez-Sanchez M, Segarra AB, Martinez-Canamero M, Prieto I. Influence of extra virgin olive oil on blood pressure and kidney angiotensinase activities in spontaneously hypertensive rats. Planta Med 81, 664-669, 2015.

Williams DP, Cash C, Rankin C, Bernardi A, Koenig J, Thayer JF. Resting heart rate variability predicts selfreported difficulties in emotion regulation: a focus on different facets of emotion regulation. Front Psychol 6, 261, 2015.

Xavier CH, Beig MI, Ianzer D, Fontes MA, Nalivaiko E. Asymmetry in the control of cardiac performance by dorsomedial hypothalamus. Am J Physiol Regul Integr Comp Physiol 304, R664-R674, 2013.

$\mathrm{Xu}$ B, Zheng H, Patel KP. Enhanced activation of RVLM-projecting PVN neurons in rats with chronic heart failure. Am J Physiol Heart Circ Physiol 302, H1700-H1711, 2012.

Zamrini EY, Meador KJ, Loring DW, Nichols FT, Lee GP, Figueroa RE, Thompson WO. Unilateral cerebral inactivation produces differential left/right heart rate responses. Neurology 40, 1408-1411, 1990.

Zhou X, Frohlich ED. Analogy of cardiac and renal complications in essential hypertension and aged SHR or L-NAME/SHR. Med Chem 3, 61-65, 2007. 